\title{
Plasmon enhancement of bulk heterojunction organic photovoltaic devices by electrode modification
}

\author{
Xiaohong Chen, ${ }^{\text {a) }}$ Chunchang Zhao, Lewis Rothberg, and Man-Kit $\mathrm{Ng}^{\mathrm{a})}$ \\ Department of Chemistry, University of Rochester, Rochester, New York 14627, USA
}

(Received 20 June 2008; accepted 31 August 2008; published online 22 September 2008)

\begin{abstract}
The efficiency of photovoltaic devices fabricated from blends of regioregular poly(3-hexylthiophene) and $\{6,6\}$-phenyl $C_{61}$-butyric acid methylester was improved by introducing a thin $\mathrm{Au}$ layer and $\mathrm{LiF}$ spacer prior to evaporation of $\mathrm{Al}$ contacts onto the polymer blend. Using an optimized $\mathrm{Au} / \mathrm{LiF} / \mathrm{Al}$ electrode gave a $20 \%-30 \%$ improved power efficiency over devices with only $\mathrm{LiF} / \mathrm{Al}$ for AM1.5 irradiation. The increased efficiency is primarily due to the increased photocurrent, and we present spectroscopic data suggesting that this can be ascribed to plasmon enhancement of the polymer absorption by nanotextured Au. (c) 2008 American Institute of Physics.

[DOI: $10.1063 / 1.2988190]$
\end{abstract}

Considerable effort has been devoted to the development of solar cells based on conjugated polymers as a promising alternative source of electrical energy. ${ }^{1-4}$ New device structures, ${ }^{2}$ materials, ${ }^{3}$ and approaches ${ }^{5}$ have been developed to improve polymer solar cell performance and devices based on bulk heterojunctions. Those formed from blended regioregular poly(3-hexylthiophene) (P3HT) and $\{6,6\}$-phenyl $C_{61}$-butyric acid methylester (PCBM) exhibit efficiencies near $5.0 \%$ with optimized device structure and fabrication conditions. ${ }^{6}$ Here we describe an electrode structure that exploits surface plasmon enhancement of the polymer absorption to improve the efficiency of P3HT:PCBM blend devices.

Excitation of surface plasmons in thin metal films has been demonstrated to enhance absorption of light of nearby molecules, ${ }^{7}$ photoluminescence, ${ }^{8}$ and Raman scattering. The phenomenon has been applied to molecular sensing, ${ }^{9}$ photovoltaic devices, ${ }^{10,11}$ and light-emitting devices. ${ }^{12}$ Additional work has demonstrated even more dramatic effects using surface plasmon resonance associated with nanotextured thin metal films and gratings that increase the efficiency of photoluminescence, ${ }^{13}$ light-emitting devices, ${ }^{14,15}$ and photodetectors. ${ }^{16}$ The effect has also been shown to be partly responsible for the enhancement of organic solar cells by the incorporation of nanotextured metal. ${ }^{17-21}$ In this letter, we report the enhancement of photocurrent and power conversion efficiency (PCE) in P3HT:PCBM bulk heterojunction photovoltaic devices utilizing surface plasmon enhancement by the incorporation of nanotextured metal into the cathode structure.

The polymer photovoltaic devices we have studied consist of a blended P3HT:PCBM thin film sandwiched between indium tin oxide (ITO) and the modified metallic cathode described below. The films were spin cast under the ambient atmosphere. A buffer layer of poly(ethylenedioxythiophene) doped with poly(styrenesulfonate) (PEDOT:PSS) of about $120 \mathrm{~nm}$ thickness was obtained by spin coating an aqueous solution (Baytron PVP Al 4083) onto ITO coated glass substrates, followed by annealing at $120^{\circ} \mathrm{C}$ for $15 \mathrm{~min}$ under mild vacuum of $10^{-2}$ Torr. Solutions containing P3HT

\footnotetext{
a) Authors to whom correspondence should be addressed. Electronic addresses: xiaohongchen@ymail.com and mankit_ng@yahoo.com.
}

and PCBM with 1:1 weight ratio dissolved in 1,2-dichlorobenzene were spin coated in air on top of the PEDOT:PSS to form $\sim 130 \mathrm{~nm}$ thick films that were also annealed at $120^{\circ} \mathrm{C}$ for 15 min under mild vacuum of $10^{-2}$ Torr. Finally, Au, LiF, and Al layers were thermally deposited onto the P3HT:PCBM blend in a chamber with base pressure $10^{-5}$ Torr to form the cathode. The active area of the devices was $0.1 \mathrm{~cm}^{2}$. Current-voltage characteristics were measured by a Keithley 2400 source-measure unit and photoexcitation provided by a $450 \mathrm{~W}$ Oriel Xe lamp with an AM1.5G filter, providing an illumination intensity of $100 \mathrm{~mW} / \mathrm{cm}^{2}$ under ambient atmospheric conditions.

Surface plasmon resonance of metallic nanoparticles is characteristic of the type of metal used and is sensitive to the sizes, shapes, and arrangements of nanoparticles as well as the surrounding environment. ${ }^{7,15,19}$ Using evaporated nanotextured metallic films, the surface plasmon resonance wavelength can be adjusted by its film thickness as illustrated for gold films in Fig. 1(a). The redshifted absorption with increasing thickness is caused by interactions between plasmons from individual gold islands as their separation is reduced and can be used to tailor the plasmon resonance to enhance the red portion of the P3HT:PCBM film absorption that contributes most to solar absorption. In the full cathode structure described above, the absorption spectra are also affected by the thickness of a LiF layer separating the $\mathrm{Au}$ and Al layers as documented by Fig. 1(b). When the gold and aluminum are in contact, surface plasmons in the gold can be dissipated efficiently and the effect of a $\mathrm{LiF}$ spacer layer is to restore the red plasmon resonance characteristic of the nanometer sized Au islands. ${ }^{22}$ The results in Fig. 1(b) suggest that it is possible for properly engineered $\mathrm{Au} / \mathrm{LiF} / \mathrm{Al}$ cathode structures to retain the desirable plasmonic features of nanotextured metals and lead to absorption and photocurrent enhancement in organic photovoltaics. The P3HT:PCBM film extinction is significantly increased by the deposition of gold [Fig. 1(c)], although this result cannot be simply interpreted as enhanced absorption by the polymers.

To investigate the effects of incorporating plasmonic properties into the cathode structure on the efficiency of polymer solar cells, we fabricated sets of eight types of devices. Figure 2 shows the current-voltage curves for the best device of each type, while Fig. 3 presents averages and stan- 


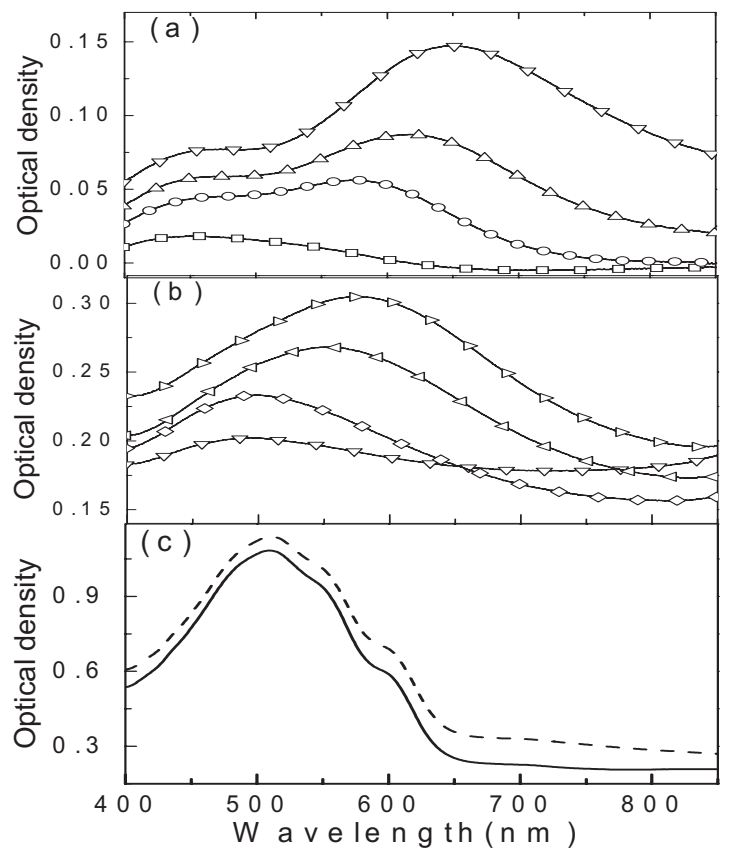

FIG. 1. Absorption spectra of thin films relevant to the photovoltaic device structures. (a) Thin films of Au with thicknesses of $2 \mathrm{~nm}(\square), 4 \mathrm{~nm}(\bigcirc), 6$ $\mathrm{nm}(\triangle)$, and $8 \mathrm{~nm}(\nabla)$. (b) Thin films consisting of Au6/Al8 $(\nabla)$, Au6/LiF1/ Al8 $(\diamond)$, Au6/LiF2.5/A18 $(\triangleleft)$, and Au6/LiF4/A18 $(\triangleright)$ where the numbers denote average layer thickness in nanometer. (c) Thin films of P3HT:PCBM (1:1) blend before (solid line) and after (dashed line) the deposition of $6 \mathrm{~nm}$ of gold.

dard deviations for the characteristics of eight devices made with each of the eight structures. Generally speaking, the variation between devices with a given structure is significantly less than the variation between devices with different structures so that we are able to make meaningful conclusions about optimal device architectures from our data. The devices with Au6/LiF1.5/Al exhibit the best performance. The short-circuit current of the worst of these devices is over $10 \%$ larger than the short-circuit current of the best performing device having a LiF/Al layer without Au. Figure 2(b) illustrates the effect of the LiF spacer by showing currentvoltage characteristics for the best device from each set

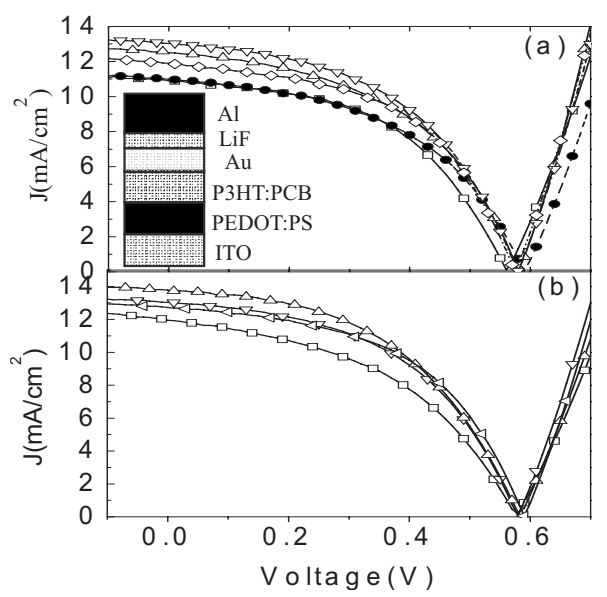

FIG. 2. Current-voltage characteristics of the best device representing each distinct structure. The cathode composition is $150 \mathrm{~nm} \mathrm{Al}$ on top of (a) LiF1.5 ( $\square)$, LiF2.5 (๑), Au3/LiF1.5 $(\triangle)$, Au6/LiF1.5 $(\nabla)$, and Au8/LiF1.5 $(\diamond)$; (b) Au6/LiF1 ( $\square$ ), Au6/LiF1.5 ( $\nabla)$, Au6/LiF3.5 ( $\triangleleft)$, and Au6/LiF2.5 $(\triangle)$. The numbers denote average layer thickness in nanometer. The inset in (a) is a schematic of device structure.

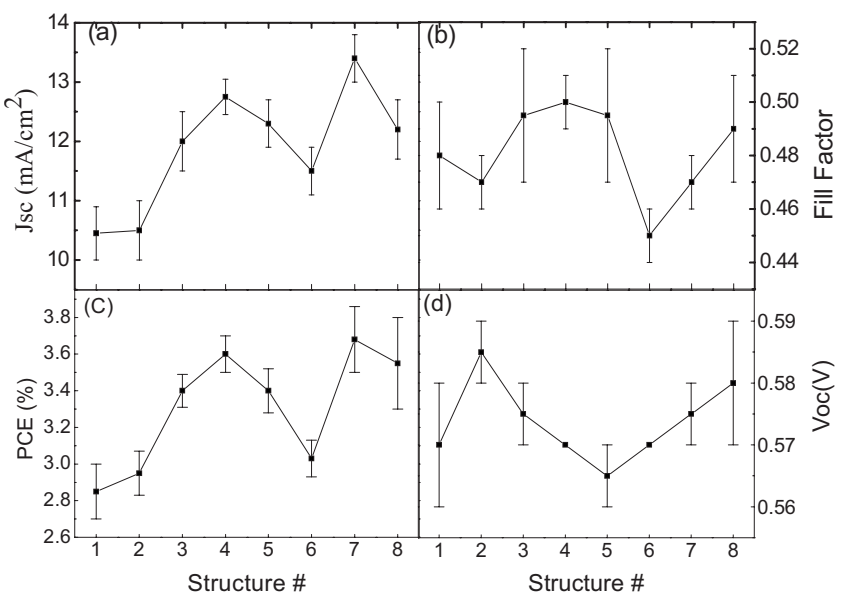

FIG. 3. Device parameters and scatter derived from eight devices of each structure. The cathode composition is $150 \mathrm{~nm} \mathrm{Al}$ on top of (1) $\mathrm{LiF} 1.5$, (2) LiF2.5, (3) Au3/LiF1.5, (4) Au6/LiF1.5, (5) Au8/LiF1.5, (6) Au6/LiF1, (7) Au6/LiF2.5, and (8) Au6/LiF3.5. The numbers denote average layer thickness in nanometer.

where the gold was $6 \mathrm{~nm}$ thick. The structure of Au6/ LiF2.5/Al has the largest short-circuit current. Consistent with our conclusions from the absorption spectra in Fig. 1, we believe that a substantial LiF spacer serves to allow the nanotextured gold film to retain strong plasmonic properties. Of course, LiF layers that are too thick serve as insulators that inhibit electron extraction at the cathode. Our data suggest that $2.5 \mathrm{~nm}$ thick layers are an optimal compromise.

As is evident from Fig. 3, the open circuit voltage and fill factor do not significantly change with increasing $\mathrm{Au}$ layer thickness. However, when we used $\mathrm{Au} / \mathrm{Al}, \mathrm{Al}$, or $\mathrm{Au}$ without $\mathrm{LiF}$ as the cathode, the open voltage and fill factor are only 0.46 and $0.40 \mathrm{~V}$, respectively. This is consistent with the observations of Brabec et al. ${ }^{23}$ who reported that solar cells with $\mathrm{LiF} / \mathrm{Al}$ or $\mathrm{Au}$ electrodes can significantly enhance open circuit voltage and fill factors relative to devices with only $\mathrm{Al}$ or $\mathrm{Au}$ electrodes. We take this to mean that the $\mathrm{LiF}$ layer in our $\mathrm{Au} / \mathrm{LiF} / \mathrm{Al}$ electrodes remains in direct contact with the P3HT:PCBM film and that the Au film exists as a discontinuous nanotextured film. Diffusion of the gold to form discrete islands is consistent with our expectations and with previous work. ${ }^{24}$ We also believe that the electrical discontinuity of the gold means that improvements due to incorporation of $\mathrm{Au}$ cannot be ascribed to changing the cathode properties.

The fact that the essential difference in the devices with incorporated gold is in enhanced photocurrent rather than effects on open circuit voltage or fill factor also argues strongly for an explanation based on plasmon-enhanced absorption by the polymers. This argument can further be strengthened by an examination of the wavelength dependence of the photocurrent. ${ }^{19}$ Figure 4(a) shows the spectrally resolved photocurrent of best devices of four types. The photocurrent in the range from 450 to $600 \mathrm{~nm}$ where Au surface plasmon resonance is strong (cf. Fig. 1) is increased substantially in the devices that have Au incorporated into the cathode structure. Figure 4(b) shows this even more clearly by plotting the photocurrent versus wavelength normalized to that of the devices without the Au film. These relative curves roughly match the extinction spectrum profiles of the Au/ $\mathrm{LiF} / \mathrm{Al}$ thin films and provide strong evidence that the plasmon enhancement of the P3HT:PCBM absorption by the 


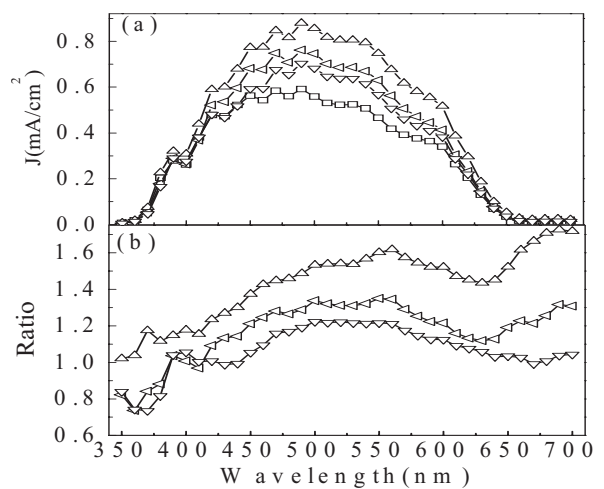

FIG. 4. (a) Spectrally resolved photocurrent for several of the device structures. The cathode composition is $150 \mathrm{~nm} \mathrm{Al}$ on top of (1) LiF1.5 ( $\square$ ), (2) Au3/LiF1.5 $(\triangleleft),(3)$ Au6/LiF1.5 $(\triangle)$, and (4) Au8/LiF1.5 ( $\nabla)$. The numbers denote average layer thickness in nanometer. (b) Photocurrent vs wavelength relative to photocurrent of the device without $\mathrm{Au}$. Curves showing the ratio of photocurrent for devices $2(\triangleleft), 3(\triangle)$, and $4(\nabla)$ divided by that for device 1.

nanotextured gold film is the root cause of the efficiency improvement of the solar cells. We also note that multiple particle plasmon resonance in gold extends to the near infrared and has the additional benefit of preferential enhancement of polymer absorption in the red to achieve better match to the solar spectrum.

In conclusion, conjugated polymer bulk heterojunction devices with suitably tailored $\mathrm{Au} / \mathrm{LiF} / \mathrm{Al}$ layered cathodes showed 20\%-30\% improved short-circuit current density and PCE over devices without gold. We interpret the changes in spectral response and effects of LiF thickness variation to mean that the introduction of a nanotextured Au thin film increases the absorption of the P3HT:PCBM thin film through plasmon-assisted localization of the electromagnetic field of the incident light. This strategy can be expected to yield direct improvements to even the highest efficiency polymer devices since it involves minimal perturbation to the morphology of the charge photogeneration layer and, judging by open circuit voltages, apparently has little effect on the contact efficiency.

The authors thank NYSTAR for financial support. L.R. is grateful for the support from NSF under Grant No. DMR0513416.

${ }^{1}$ H. Hoppe and N. S. Sariciftci, J. Mater. Res. 19, 1924 (2004).

${ }^{2}$ J. Y. Kim, K. Lee, N. E. Coates, D. Moses, T. Q. Nguyen, M. Dante, and A. J. Heeger, Science 317, 222 (2007).

${ }^{3}$ W. Y. Wong, X. Z. Wang, Z. He, A. B. Djurisic, C. T. Yip, K. Y. Cheung, H. Wang, C. S. K. Mak, and W. K. Chan, Nat. Mater. 6, 521 (2007).

${ }^{4}$ C. Zhao, X. Chen, Y. Zhang, and M. Ng, J. Polym. Sci., Part A: Polym. Chem. 46, 2680 (2008)

${ }^{5}$ G. Li, Y. Yao, H. Yang, V. Shrotriya, G. Yang, and Y. Yang, Adv. Funct. Mater. 17, 1636 (2007).

${ }^{6}$ C. J. Ko, Y. K. Lin, F. C. Chen, and C. W. Chu, Appl. Phys. Lett. 90, 063509 (2007).

${ }^{7}$ D. M. Schaadt, B. Feng, and E. T. Yu, Appl. Phys. Lett. 86, 063106 (2005).

${ }^{8}$ J. R. Lakowicz, Anal. Biochem. 337, 171 (2005).

${ }^{9}$ T. A. Taton, C. A. Mirkin, and R. L. Letsinger, Science 289, 1757 (2000).

${ }^{10}$ B. P. Rand, P. Peumans, and S. R. Forrest, J. Appl. Phys. 96, 7519 (2004).

${ }^{11}$ K. Kim and D. L. Carroll, Appl. Phys. Lett. 87, 203113 (2005).

${ }^{12}$ H. J. Park, D. Vak, Y. Y. Noh, B. Lim, and D. Y. Kim, Appl. Phys. Lett. 90, 161107 (2007).

${ }^{13}$ S. Pan and L. J. Rothberg, J. Am. Chem. Soc. 127, 6087 (2005).

${ }^{14}$ D. K. Gifford and D. G. Hall, Appl. Phys. Lett. 81, 4315 (2002).

${ }^{15}$ K. Okamoto, I. Niki, A. Shvartser, Y. Narukawa, T. Mukai, and A. Scherer, Nat. Mater. 3, 601 (2004).

${ }^{16}$ H. R. Stuart and D. G. Hall, Appl. Phys. Lett. 69, 2327 (1996).

${ }^{17}$ T. Wakamatsu, K. Saito, Y. Sakakibara, and H. Yokoyama, Jpn. J. Appl. Phys., Part 1 36, 155 (1997).

${ }^{18}$ K. Tvingstedt, N. K. Persson, O. Inganäs, A. Rahachou, and I. V. Zozoulenko, Appl. Phys. Lett. 91, 113514 (2007).

${ }^{19}$ S. Pan and L. J. Rothberg, Proc. SPIE 6641, 664109 (2007).

${ }^{20}$ A. J. Morfa, K. L. Rowlen, T. H. Reilly, M. J. Romero, and J. van de Lagemaat, Appl. Phys. Lett. 92, 013504 (2008).

${ }^{21}$ T. H. Reilly, J. van de Lagemaat, R. C. Tenent, A. J. Morfa, and K. L. Rowlen, Appl. Phys. Lett. 92, 243304 (2008).

${ }^{22}$ Nanophotonics with Surface Plasmons, edited by V. M. Shalaev and S. Kawata (Elsevier, New York, 2007).

${ }^{23}$ C. J. Brabec, S. E. Shaheen, C. Winder, N. S. Sariciftci, and P. Denk, Appl. Phys. Lett. 80, 1288 (2002).

${ }^{24}$ I. Doron-Mor, Z. Barkay, N. Filip-Granit, A. Vaskevich, and I. Rubinstein, Chem. Mater. 16, 3476 (2004). 